\title{
Plasma and urine metabolite profiling reveals the protective effect of Clinacanthus nutans in an ovalbumin-induced anaphylaxis model: ${ }^{1} \mathrm{H}-\mathrm{NMR}$ metabolomics approach
}

\begin{abstract}
The present study sought to identify the key biomarkers and pathways involved in the induction of allergic sensitization to ovalbumin and to elucidate the potential anti-anaphylaxis property of Clinacanthus nutans (Burm. f.) Lindau water leaf extract, a Southeast Asia herb in an in vivo ovalbumin-induced active systemic anaphylaxis model evaluated by $1 \mathrm{H}-\mathrm{NMR}$ metabolomics. The results revealed that carbohydrate metabolism (glucose, myo-inositol, galactarate) and lipid metabolism (glycerol, choline, sn-glycero-3-phosphocholine) are the key requisites for the induction of anaphylaxis reaction. Sensitized rats treated with $2000 \mathrm{mg} / \mathrm{kg}$ bw C. nutans extract before ovalbumin challenge showed a positive correlation with the normal group and was negatively related to the induced group. Further $1 \mathrm{H}-\mathrm{NMR}$ analysis in complement with Kyoto Encyclopedia of Genes and Genomes (KEGG) reveals the protective effect of C. nutans extract against ovalbumin-induced anaphylaxis through the down-regulation of lipid metabolism (choline, sn-glycero-3-phosphocholine), carbohydrate and signal transduction system (glucose, myo-inositol, galactarate) and up-regulation of citrate cycle intermediates (citrate, 2-oxoglutarate, succinate), propanoate metabolism (1,2-propanediol), amino acid metabolism (betaine, N,N-dimethylglycine, methylguanidine, valine) and nucleotide metabolism (malonate, allantoin). In summary, this study reports for the first time, C. nutans water extract is a potential anti-anaphylactic agent and 1H-NMR metabolomics is a great alternative analytical tool to explicate the mechanism of action of anaphylaxis.
\end{abstract}

Keyword: Clinacanthus nutans; Medicinal plant; Ovalbumin; Active systemic anaphylaxis; NMR metabolomics; Multivariate analysis 